\title{
The knitting community-based trial for older women with osteoarthritis of the hands: design and rationale of a randomized controlled trial
}

Paulette Guitard ${ }^{*}$, Lucie Brosseau', George A. Wells ${ }^{2}$, Nicole Paquet ${ }^{1}$, Gail Paterson ${ }^{3}$, Karine Toupin-April ${ }^{4}$, Sabrina Cavallo ${ }^{5}$, Sibel Z. Aydin ${ }^{6}$, Guillaume Léonard ${ }^{7}$ and Gino De Angelis ${ }^{1}$

\begin{abstract}
Background: The prevalence of hand osteoarthritis $(\mathrm{HOA})$ has been reported to be higher amongst women over 50 years old (66\%) compared to men of the same age (34\%). Although exercise therapy has been shown effective in reducing symptoms and disability associated with $\mathrm{HOA}$, adherence to treatment programs remains low. The primary objective of this RCT is to examine the effectiveness of a 12-week knitting program for morning stiffness (primary outcome) and pain relief (secondary outcome) $2 \mathrm{~h}$ post-wakening in females (aged 50 to 85 years old) with mild to moderate hand osteoarthritis ( $\mathrm{HOA})$.

Methods/design: A single-blind, two-arm randomized controlled trial (RCT) with a parallel group design will be used to reach this objective and compare results to a control group receiving an educational pamphlet on osteoarththritis $(\mathrm{OA})$ designed by the Arthritis Society. The premise behind the knitting program is to use a meaningful occupation as the main component of an exercise program. The knitting program will include two components: 1) bi-weekly 20-min knitting sessions at a senior's club and 2) 20-min home daily knitting sessions for the five remaining weekdays. Participants assigned to the control group will be encouraged to read the educational pamphlet and continue with usual routine. Pain, morning stiffness, hand function, self-efficacy and quality of life will be measured at baseline, six weeks, 12 weeks (end of program) with standardized tools. We hypothesize that participants in the knitting program will have significant improvements in all clinical outcomes compared to the control group.

A published case study as well as the preliminary results of a feasibility study as examined through a 6-week pre-post study ( $n=5$ women with HOA) involving 20-min daily knitting morning sessions led to this proposed randomized controlled trial research protocol. This article describes the intervention, the empirical evidence to support it.
\end{abstract}

Discussion: This knitting RCT has the potential to enhance our understanding of the daily HOA symptoms control and exercise adherence, refine functional exercise recommendations in this prevalent disease, and reduce the burden of disability in older women.

Trial Registration: (ACTRN12617000843358) registered on 7/06/2017.

\footnotetext{
* Correspondence: Guitardp@uottawa.ca

${ }^{1}$ School of Rehabilitation Sciences, Faculty of Health Sciences, University of

Ottawa, 451 Smyth Road, Ottawa, ON K1H 8M5, Canada

Full list of author information is available at the end of the article
} 


\section{Background}

Osteoarthritis affects a large proportion of the world population and hand osteoarthritis (HOA) represents the highest age-standardized total prevalence (43.3\%) in terms of joint site compared to hip OA (23.9\%) and knee OA (10.9\%) [1]. A higher age-standardized total prevalence of HOA is observed amongst women over 50 years old $(65.8 \%)$ compared to men of the same age (34.2\%) [2]. The prevalence of HOA is expected to increase with the aging of the population [3]. HOA is recognized as a significant cause of disability resulting in activity and participation limitations as well as reduced quality of life (QoL) [4, 5] due to presence of pain, morning stiffness, tenderness and swelling of the fingers joints, diminished grip strength and psychological problems $[2,6]$.

Active hand exercises (i.e. strengthening exercises and yoga) have been shown to be an effective strategy to reduce morning stiffness $[7,8]$ and pain [9-11], increase range of motion [12, 13] grip and/or pinch strength [7-9, 11-16] improve hand functional status in $\operatorname{HOA}[8,10]$ and reduce disease activity [7]. To our knowledge, no studies examined the effect of functional activities in the management of HOA. Several authors suggested that there is an urgent need for more trials of nonpharmacological and nonsurgical interventions for HOA [8, 17-20].

Isometric contractions (i.e. a type of muscle contraction involved knitting) have been shown effective to reduce pain perception, both in healthy individuals and in patients suffering from chronic pain [21, 22]. For healthy individuals, long duration activity (30 $\mathrm{min})$ seems to be necessary to trigger exercise-induced hypoalgesia [22, 23]. For patients with chronic pain, meaningful change in pain is observed both for high and low intensity exercises protocols [21, 24, 25]. Psychosocial aspects can also contribute to the beneficial effects of exercise on pain [22, 26, 27].

According to the Canadian Model of Occupational Performance and Engagement (CMOP-E), [28] occupations bring meaning to life. Occupational therapy explores the therapeutic potential of occupations; it is believed that the power and the positive effects of occupations are greater when client can choose, control and get a sense of accomplishment through them. The movements typically incorporated in hand therapy for HOA (finger exercises [9] and muscle activation [22, 23] are also present in knitting. Knitting can be considered a purposeful and meaningful occupation. As such, knitting may be more appealing and meaningful for elderly women to participate in compared to regimented exercises programs. This study embeds the functional activity of knitting in an exercise program that may demonstrate greater adherence than regimented hand exercises [29]. Knitting also represents a promising activity for older individuals suffering from HOA pain via its potential effect on psychological and social factors (e.g. positive effect on mood and social isolation, particularly if knitting is done at a senior's club). Indeed, it could be more appealing, motivating, enjoyable and rewarding to perform an activity that is highly appreciated [30] than a series of prescribed therapeutic exercise. To our knowledge, no studies have examined the effect of functional activities in the management of HOA.

In this research protocol, knitting is used in a larger context. It is prescribed and is structured in terms of frequency and duration, but not regarding intensity, style of knitting or needles holding (respect of personal fashion and willingness)) and is a replacement of a traditional prescribed exercise program during a therapy. A therapeutic aim would be to prevent morning stiffness as much as possible to maintain a regular level of daily activities. The proposed randomized controlled trial (RCT) will incorporate meaningful occupation (i.e. knitting) within a structured and supervised context.

The results of a recent case study (Brosseau \& Leonard, 2017) revealed that a 12-week low intensity knitting program is a promising self-management strategy for mild to moderate HOA. Performing daily early morning knitting over 12 weeks resulted in a 50\% short-term improvement in daily pain and joint stiffness relief in bilateral osteoarthritic fingers of an 86-year old woman who had been living with the disease for at least 40 years. She showed strong adherence to the program by participating in all knitting sessions (100\%). This individual reported improved patient global assessment [31], strong goal attainment [32], and improved self-efficacy in managing her arthritis pain (60\% improvement) [33].

Following the results of this case study, a feasibility study was conducted. The preliminary results showed also that the five elderly women ( 80 to 87 years old) had a $100 \%$ adherence rate during a 6 weeks knitting intervention. The six-week knitting intervention, the selected measurements and the adapted logbook were deemed useful, easy to follow and were well-accepted by older women with HOA. Furthermore, the five participants reported an average daily immediate relief of $45 \%$ and $77 \%$ respectfully for pain and morning stiffness (i.e. great effect on morning stiffness). These daily improvements were maintained over two as well as four consecutive hours. Four of five participants loved to knit and had knitted in the past, but had not knitted for at least two months. The fifth participant never knitted in her life. She did not enjoy it, but nevertheless adhered to the program until the completion of the study.

The proposed knitting program is based on: 1) evidence stemming from physiological studies [34-36]; 2) recommendations for individuals over 50 years old with OA [37] and specifically with HOA [12]; 3) evidencebased general exercise protocols for $\operatorname{HOA}[5,38,39]$ and 
OA of the thumb [40-42]; 4) current RCTs on therapeutic exercises for HOA $[9,14]$ and on the case study parameters described above [43]. In the proposed study, knitting will be a low-intensity hand exercise therapy comprising bilaterally dynamic and isometric movement of fingers, thumbs and wrists. Despite knitting being endorsed by The Arthritis Foundation [44] devoted to arthritis as a therapeutic activity for adults living with $\mathrm{HOA}$, to our knowledge, no other comparative study studied its effectiveness on reducing symptoms related to HOA, potentially decreasing their impact in daily life and thus improving quality of life.

The primary objective of this RCT will be to examine the effectiveness of a 12-week knitting program for morning stiffness (primary outcome) and pain relief (secondary outcome) for participants in the knitting in Group 1 compared to participants in the control group assigned to a waiting list (Group 2) in older females (aged 50 to 85 years old) with mild to moderate osteoarthritis of the hands (HOA) after $2 \mathrm{~h}$ post-awakening. The secondary objective is to determine if older females with mild to moderate HOA using an adapted knitting program (Group 1) have improved hand and finger strength and physical function, self-efficacy, hand physical activity level and adherence, global patient improvement as well as quality of life (QoL) compared to a waiting list control group (Group 2) over 12 weeks of intervention and after a follow-up at 4 weeks post intervention. Our general hypothesis is that older females with mild to moderate HOA in the 12-week knitting group (Group 1) will have significant improvements in all daily clinical and implementation outcomes compared to the participants in the waiting list (Group 2) after 12 weeks of intervention.

\section{Methods/design}

The following methodology is in full agreement with the SPIRIT (Standard Protocol Items for Randomized Trials) recommendations [45-47] and the Osteoarthritis Research Society International (OARSI) recommendations for RCTs [48] to ensure methodological rigor. Consolidated Standards of Reporting Trials (CONSORT) [49] guidelines will be followed for reporting on the results in a subsequent article.

\section{Study design}

The study is a single blind, two-arm RCT with a parallel group design to compare two study groups: 1) a knitting intervention (Group 1) and 2) a waiting list control group (Group 2). The intervention period will be 12 weeks plus a follow-up at 4 weeks post-intervention to measure the retention effect. Since this RCT involves a physical intervention (i.e. knitting program), the therapist, participants, and research coordinator administering the program will not be blinded. A blinded independent assessor will be trained to assess the participants though performance evaluation and self-reported questionnaires given at baseline, 3,6,9,12-week as well as at 4-week follow-up to reduce detection bias. With training and standard operating procedures, it is anticipated that any performance bias due to unblinding will be minimized. The study design flow chart is presented in Fig. 1. The study protocol is approved by the University of Ottawa Research Ethics Board (\#H02-16-12).

\section{Study population}

The diagnosis of HOA will be made by a rheumatologist (SZA) and will be consistent with the clinical criteria as set out by the American College of Rheumatology (ACR) classification of HOA [50], the radiologic criteria according to Kallman et al. [51] (1989), and the disease activity criteria according to the Doyle Articular Index [52]. The severity of the HOA will be determined according to ACR classification of HOA and by clinically assessing the presence of (1) pain, aching or joint stiffness, (2) bony enlargement of 2 or more of 10 selected finger joints, (3) bony enlargement of at least a distal interphalageal (DIP) joint, and (4) fewer than 2 swollen metacarpophalageal (MCP) joints, or (5) deformity of at least 1 of 10 selected joints. Presence of OA will be confirmed by a recent X-rays taken of each hand (less than 1 year); films will be interpreted by a radiologist and reports will be sent to the rheumatologist (SZA) to confirm diagnosis and classification.

\section{Inclusion/exclusion criteria}

To be eligible for this RCT, participants will be required to: 1) meet the ACR clinical and radiographic criteria of definite HOA and of mild to moderate severity status [50] and have experienced pain symptoms for at least 3 months, 2) be between 50 and 85 years of age, 3) have a level of morning stiffness of at least $4 / 10$ on a visual analogue scale (VAS) [53] at the time of study entry, 4) display X-ray evidence of joint space narrowing of the hands [51], 5) have no knowledge of knitting (do not know how to knit) or have not knitted in the last 2 months; 6 ) be available for sessions at the senior's club twice a week, 7) be able to understand written and verbal English instructions. Participants will be excluded if they: 1) are current active knitters; 2) are unwilling or unable to adhere to the knitting program for 12 consecutive weeks, 3) have other orthopedic, rheumatologic diseases (i.e. inflammatory arthritis, psoriasis arthritis, gout...), or evidence of chondrocalcinosis, 4) had any prior surgery for the finger joints, 5) have any acute disease, such as uncontrolled diabetes mellitus, untreated hypertension, neurological deficits (motor or sensory) or cognitive deficit and mental health conditions, 6) are taking OA medication that is expected to change during 


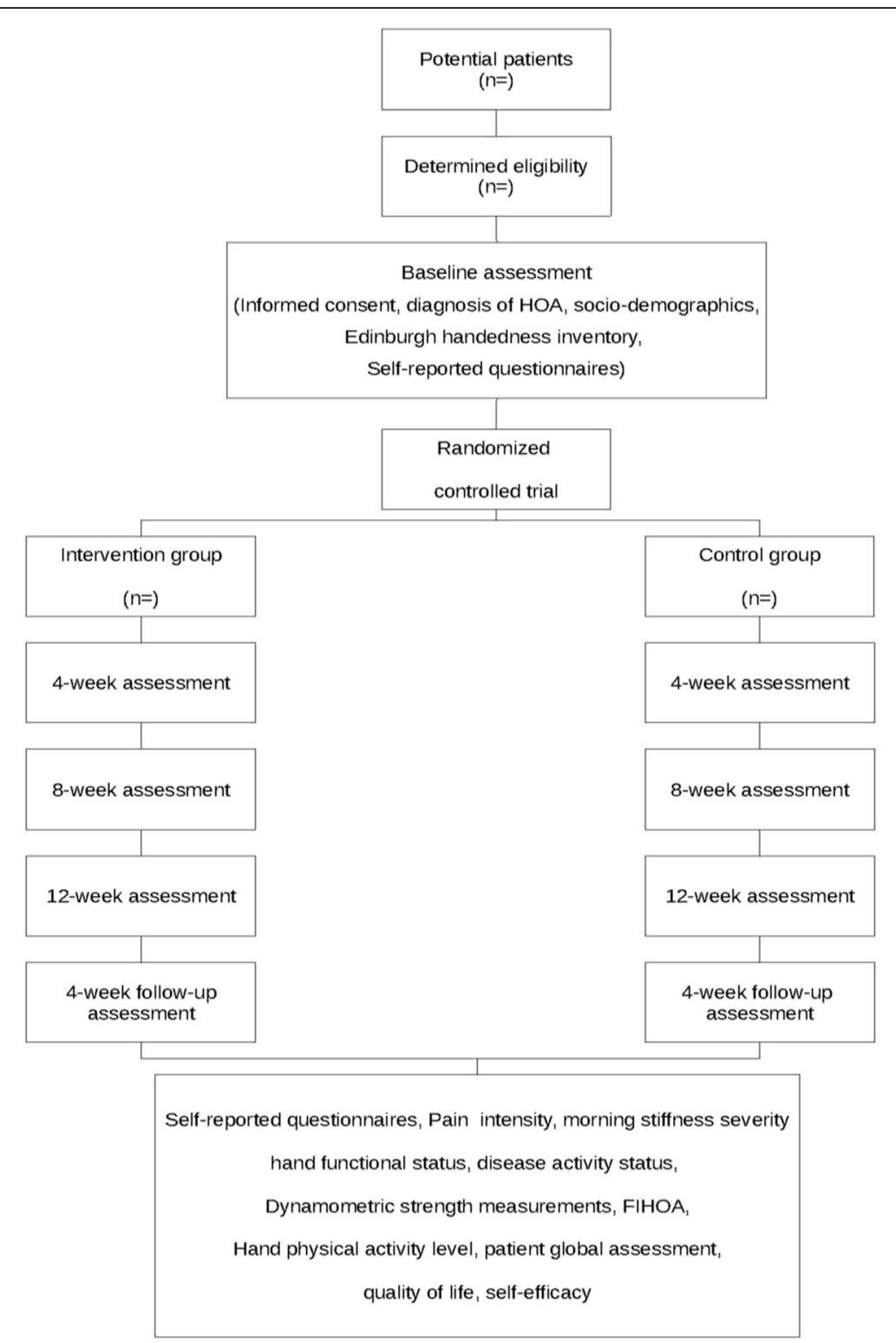

Fig. 1 Study design flow chart

the study period, 7) receiving current rehabilitation treatment or any other pain-related treatment besides medication for OA, 8) have received corticosteroid injection of finger joints within the last 6 months, 9) plan to move or relocate within 6 months.

\section{Recruitment}

Three main methods will be used to recruit potential participants. Advertisements in local newspapers will be placed. Information letters will be sent to local rheumatologists and posters will be placed in waiting rooms of each different Ottawa-based rheumatology units and TAS office. Potential participants will be invited to complete an online eligibility/admission questionnaire
[53], including sociodemographic, initial intensity, location (e.g. finger joints versus thumb joints; right versus left hand), timing, intensity and duration of morning stiffness and pain [54-56] and handedness information $[57,58]$ to ensure that they meet the study's selection criteria prior to randomization. If deemed eligible, participants will be invited to meet the research coordinator to confirm eligibility, sign an informed consent form, and complete baseline evaluations. In a previous RCT on HOA [59], 40 participants over 3 months were recruited, thus the data collection involving a 12-week knitting program for each participant should represent approximately six additional months, leaving 2 months for data analysis and writing the scientific report. 


\section{Group assignment}

Eligible participants will be randomly assigned to each group (ratio1:1). Participants of group 2 will have access to the knitting program after the completion of the RCT. Central randomization [60] based on a sequence of computer-generated random numbers (using statistical software SAS macro in SAS 9.3) using a blocking factor (randomly varying between 4 and 6 ) will be used.

The research coordinator, who is not involved in data collection, will contact the research study Methods Center data manager. Prior to running the randomization program, the data manager will document the participant's initials (first and last) and date of birth (month and year). After running the program, the data manager will document the intervention assignment with the participant information, assign a study identification (ID) and then inform the research assistant of the assignment and participant ID. This process will help ensure concealment of allocation. After randomization, the participant will be informed of their group assignment.

\section{Intervention}

Participants in both groups will continue with their ongoing medical care To measure the true effect of the knitting intervention, active hand exercises need to be avoided since they are proven effective for HOA [7, 9-16, 61]. This may not be possible thus all participants will complete a daily activity log.

Participants in Group 1 will take part in a lowintensity knitting program, performed as a morning functional activity, which will comprise two components: 1) bi-weekly 20-min knitting sessions at a senior's club and 2) 20-min home daily knitting sessions for the five remaining weekdays over 12 consecutive weeks (total of 90 sessions). The bi-weekly 20-min knitting session will take place, at a Senior's Club in metropolitan Ottawa, on Tuesday and Thursday mornings with a group of regular members who knit wool blankets for sick children admitted CHEO, a regional Children's Hospital. The blankets are composed of individual squares that are assembled together. Each wool square takes approximately $20 \mathrm{~min}$ for a beginner knitter. Each knitter will be encouraged to knit one wool square per day for a total of seven squares per week. To avoid excessive isometric strength and potential muscle fatigue with the use of small needles that require precise pinching, participants will use specific knitting needle and wool sizes (sizes \#6 and \#5 respectively according to the Standard Yarn Weight System (http://www.craftyarncouncil.com/ weight.html). Study participants will learn how to knit with a qualified instructor from Senior's Club during the knitting sessions which will also be supervised by a trained OT to ensure that the physiological and clinical characteristics of therapeutic exercise are being met. A knitting instructor will ensure that participants follow the prescribed program and if their individual logbooks, where they recorded their daily hand activities and morning stiffness and pain levels, are filled properly. In the event that a session is missed, participants in Group 1 will receive a telephone reminder from the research assistant to encourage them to attend the next session. At the end of 12 weeks, participants will be encouraged to continue on with the program if they gained an improvement of their condition.

Participants assigned to the control group (Group 2) will be placed on a waiting list until the end of the study (12 weeks) plus the 4-week follow-up period. The research coordinator will offer one introductory session to explain how to record any activity in their logbooks as well a weekly telephone follow-up by the coordinator to if participant understood well how to fill them daily. Participants will not be permitted to attend knitting sessions at the senior's club during the study duration. After the completion of the study duration, the knitting sessions will be available to all study participants. To avoid potential contamination, individuals in Group 2 will have no contact with the individuals registered at Senior's Club in Group 1. Club membership annual fees (\$10/year) will be paid for all study participants in Group 1 and for those in Group 2 only after the completion of the study. Free knitting material and lessons will be provided for all study participants.

\section{Self-reported and performance-based clinical outcomes outcome measures (Table 1)}

The clinical outcomes were selected according to the OMERACT framework illustrated in Fig. 2 and include the followings:

\section{Self-reported clinical outcomes (Table 1)}

Morning stiffness and pain relief, using daily visual analogue scale for morning stiffness (primary outcome) and for pain (secondary outcome) will be recorded before (baseline) and after knitting (immediately, $2 \mathrm{~h}$ and 4 hours after the 20-min knitting daily morning activity). Daily morning stiffness after $2 \mathrm{~h}$ knitting daily morning activity will be our primary outcome measure of this protocol. This choice of primary and intermediate outcomes is based on the feasibility study conducted by the team.

Functional status, using Australian/Canadian Osteoarthritis Hand Index (AUSCAN) tool as a validated, reliable and responsive measurement scale for HOA [62-64] was adopted. The AUSCAN includes a 15-item scale, with items grouped into three sub-sections: A) pain intensity, B) stiffness severity and C) hand functional status/difficulty in activities of daily living. It uses a five-point scoring system. A score of 0 represents no pain or no severity in stiffness or no difficulty in performing functional tasks; 
Table 1 Assessment schedule for primary, and secondary outcome measures

\begin{tabular}{|c|c|c|c|c|c|c|}
\hline Assessment & Admission & Daily & Baseline & $\begin{array}{l}3 / 6 / 9 \text { weeks mid } \\
\text { intervention }\end{array}$ & $\begin{array}{l}12 \text { weeks end } \\
\text { of intervention }\end{array}$ & $\begin{array}{l}4 \text { weeks } \\
\text { follow-up }\end{array}$ \\
\hline Informed consent (pre-admission) & $x$ & & & & & \\
\hline Diagnosis of HOA based on ACR criteria & $x$ & & & & & \\
\hline Socio-demographics & $x$ & & & & & \\
\hline Edinburgh Handedness Inventory & $x$ & & & & & \\
\hline $\begin{array}{l}\text { Self-reported daily morning stiffness intensity } \\
\text { (Visual analogue scale: VAS) (primary daily outcome) }\end{array}$ & $x$ & $x$ & $x$ & $x$ & $x$ & $x$ \\
\hline $\begin{array}{l}\text { Self-reported daily pain intensity (VAS) } \\
\text { (intermediate daily outcome) }\end{array}$ & $x$ & $x$ & $x$ & $x$ & $x$ & $x$ \\
\hline $\begin{array}{l}\text { Pain intensity (Auscan) } \\
\text { (periodic secondary measurement) }\end{array}$ & & & $x$ & $x$ & $x$ & $x$ \\
\hline $\begin{array}{l}\text { Morning stiffness severity (Auscan) } \\
\text { (periodic secondary outcome) }\end{array}$ & & & $x$ & $x$ & $x$ & $x$ \\
\hline $\begin{array}{l}\text { Hand functional status/difficulty in activities of daily } \\
\text { living (Auscan) (periodic secondary outcome) }\end{array}$ & & & $x$ & $x$ & $x$ & $x$ \\
\hline Disease activity status (periodic secondary outcome) & & & $x$ & $x$ & $x$ & $x$ \\
\hline $\begin{array}{l}\text { Dynamometric strength measurements (Grip and pinch } \\
\text { measurements) (periodic secondary outcome) }\end{array}$ & & & $x$ & $x$ & $x$ & $x$ \\
\hline $\begin{array}{l}\text { The Functional Index for Hand Osteoarthritis (FIHOA) } \\
\text { (periodic secondary outcome) }\end{array}$ & & & $x$ & $x$ & $x$ & $x$ \\
\hline Hand physical activity level (periodic secondary outcome) & & $x$ & $x$ & $x$ & $x$ & $x$ \\
\hline Patient global assessment (periodic secondary outcome) & & & $x$ & $x$ & $x$ & $x$ \\
\hline Quality of life (EQ-5D) (periodic secondary outcome) & & & $x$ & $x$ & $x$ & $x$ \\
\hline Self-efficacy (periodic secondary outcome) & & & $x$ & $x$ & $x$ & $x$ \\
\hline $\begin{array}{l}\text { Physical Activity Enjoyment Scale } \\
\text { (PACES) (Group } 1 \text { only) }\end{array}$ & & & & $x$ & $x$ & $x$ \\
\hline $\begin{array}{l}\text { Adherence to knitting program } \\
\text { (Daily secondary outcome) }\end{array}$ & & & $x$ & $x$ & $x$ & $x$ \\
\hline 7-day Physical Activity Recall (PAR) (Daily secondary outcome) & & & $x$ & $x$ & $x$ & $X$ \\
\hline
\end{tabular}

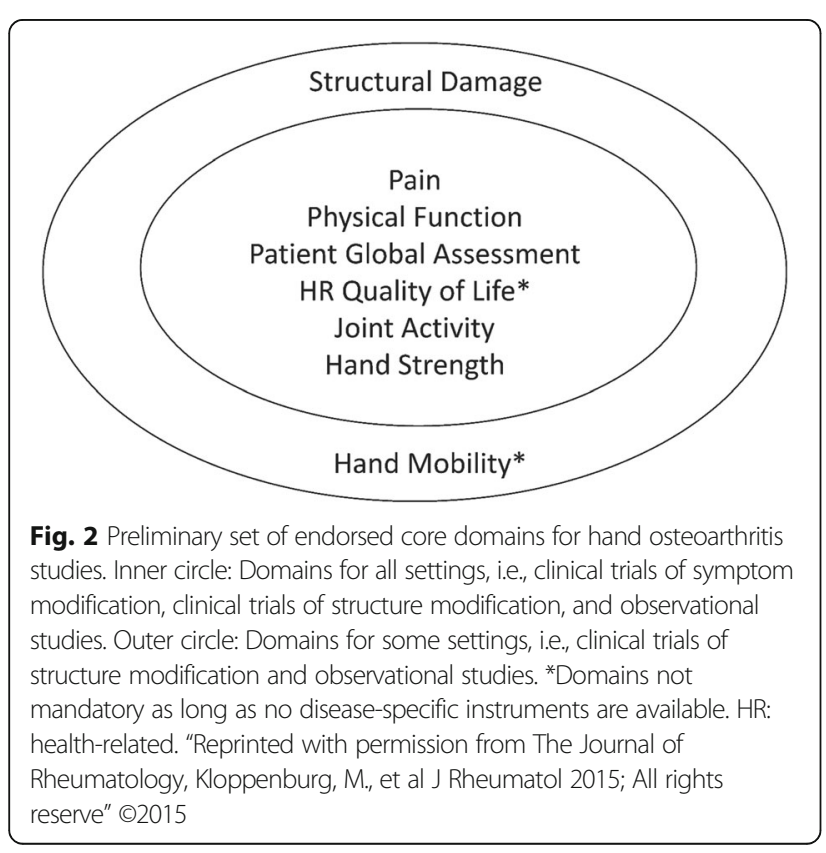

scores between one and four represent mild to extreme gradations, respectively. Morning stiffness and hand functional status are included in the AUSCAN.

Hand activity level, using an adapted 7-Day Physical Activity Readiness (PAR) [65-67] was used. The 7-day PAR is a validated instrument in a calendar format in which a participant can indicate the PA duration (minutes per day) and PA frequency (days per week). An adapted version of the 7-day PAR will be included in the logbooks where physical activity is split into distinct Knitting and other hand activities categories. The logbooks will also include a daily visual analogue scale for morning stiffness and pain intensity before and after knitting, information about hand physical activity level and knitting technique as well as a weekly questionnaire on actual changes in PA, medication intake (not encouraged), habits and adverse events. A similar logbook was created for PA activity other than knitting recording for the participants in the control group 2. Patient global assessment will be evaluated by asking patients if their condition after knitting program "fully improved", 
"partially improved", "did not improve" or "was worse compared to the beginning of the study" [31]. Health-related QoL will be assessed using the EuroQoL Index (EQ5D-5 L) [68] including mobility, self-care, usual activities, pain/discomfort and anxiety/depression subscales.

\section{Performance-based clinical outcomes (Table 1)}

Additionally, the disease activity status will measure the presence of finger inflammation of active joints using a standardized physical assessment.

(https://www.arthritis.ca/healthcare-professionals/standardized-assessment-of-joint-inflammation-(saj) featured by TAS and performed by a therapist from TAS. Two physical assessments will also be performed: 1) The Functional Index for Hand Osteoarthritis (FIHOA) [69] will also be used as a performance-based physical function measure as well as 2) dynamometric strength measurements for hand/fingers [40].

\section{Implementation outcomes}

The following implementation outcomes are not represented in the OMERACT framework (fig. 2). Self-efficacy will be assessed using The Stanford Arthritis SelfEfficacy Scale (ASES) [33], a ten-point scale ranging from one (very uncertain) to 10 (very certain). Level of knitting enjoyment will be measured using Physical Activity Enjoyment Scale (PACES) for participant in group 1 only [70]. Adherence to PA/knitting program will be estimated as the number of knitting sessions attended at the Club and performed at home divided by the number of knitting sessions prescribed (84 sessions), as recorded in the participants' logbooks, using the 7Day PAR calendar [65] as well as attendance at the senior's club. The number of wool squares that were knitted will be also reported in the participants' logbooks. However, knitting technique will be recorded how the participants hold the stabilizing and mobile needle and which hand is used by the trained OT. Adverse events observed will be also compiled. All these clinical and implementation outcomes measures were pilot tested [43].

Please insert Table 1 Assessment schedule for primary, and secondary outcome measures.

\section{Sample size calculation}

The most relevant information on the standard deviation available was from the feasibility study. Even though the sample of this feasibility was small $(n=5)$, the participants and the environment in which the study was conducted and the measurements taken are directly in alignment with that for the proposed RCT. Each patient was assessed at $2 \mathrm{~h}$. The reported Area Under the Curve (AUC) score, as a proportion of the 42 day potential AUC score of 420 (42 days with maximum score of 10; $10 \mathrm{~cm}$ Visual Analogue Scale (VAS) [53], at $2 \mathrm{~h}$ post had a mean of 0.188 and standard deviation of $0.261 \mathrm{~cm}$, based on the observed AUC scores (i.e. mean and standard deviation of the 5 AUC scores of the participants were calculated). The large effect size of 0.8 was determined based on discussions among the study investigators and other experts in the field. A large effect size was believed to be needed since a substantive morning stiffness improvement would be required to lead to potentially important improvements in subsequent daily functioning; whereas smaller changes in effect size were not believed to be substantive enough to impact these activities of daily functioning. To detect an effect size of 0.8 , with power of $80 \%$ and level of significance of 0.05 , a sample size of 28 participants per group will be required. No dropouts in the pilot were experienced and none are expected in the main study given the short duration of the study; however a $5 \%$ dropout is assumed and a sample size of 30 per group will be recruited.

No dropouts in the feasibility study occurred and none are expected in the main study given the short duration of the study; however a $5 \%$ dropout is assumed and a sample size of 30 per group will be recruited.

\section{Measurement frequency}

Clinical and implementation evaluations will be performed by the independent and trained research therapist at baseline, 3 weeks, 6 weeks, 9 weeks, and end of study $(12$ weeks $)+4$ weeks FU for participants in both groups. Measurement instruments will be calibrated every month before the evaluation sessions and will be administered in a randomized sequence. Independent evaluation sheets will be used for each patient at each assessment session in order to minimize recording bias. All measurements will be performed through the use of electronic self-reported questionnaires on a laptop using the survey website. The evaluations will take approximately $60 \mathrm{~min}$ to complete and will be performed in a private assessment room located at University of Ottawa to optimize blinding of the evaluator.

\section{Statistical analysis}

Descriptive statistics will be used to summarize the study variables and to assess the distributional assumptions of the statistical techniques used. An intention to treat analysis will be conducted for all the data analyses.

A per protocol analysis will be considered as part of the sensitivity analyses. The primary outcome is the area under the curve (AUC) at $2 \mathrm{~h}$ for stiffness. That is, stiffness at $2 \mathrm{~h}$ post-awakening from sleep will be assessed each day for 12 weeks, and the AUC over this 12 week period will then be calculated. Similar AUCs will be calculated for stiffness at $0 \mathrm{~h}$ (at awakening), $4 \mathrm{~h}$ (postawakening) and evening. Also, similar AUCs for the secondary outcomes pain and function will be calculated. 
For the primary outcome (AUC at $2 \mathrm{~h}$ for stiffness) participants in the knitting group will be compared to participants in the control group using independent Student's t-test with the pooled or separate variance estimate as appropriate. For the primary and secondary outcomes, the area under the curve (AUC) for stiffness, pain and function will be compared using a 2-way analysis of variance (ANOVA) with the between factor group (knitting vs control) and within factor time $(0 \mathrm{~h}$, $2 \mathrm{~h}, 4 \mathrm{~h}$, evening). Tukey's [71] honest significance difference (HSD) will be used to make specific pairwise comparisons. The secondary outcomes, AUSCAN overall, AUSCAN pain, AUSCAN stiffness, AUSCAN function, hand and fingers strength and physical function, selfefficacy, hand physical activity level and adherence, global patient improvement as well as QoL will be compared using a 2-way analysis of variance (ANOVA) with the between factor group (knitting vs control) and within factor time (baseline, 3 wk., 6 wk., 9 wk., 12 wk. and 4-wk Follow-Up). Again, Tukey's HSD [71] will be used to make specific pairwise comparisons. As a secondary analysis, additional time assessments to 16 weeks will be included in the above analyses to assess followup. An alpha significance of 0.05 was chosen for all analyses. Mixed models repeated measured will be used to accommodate missing data. No interim efficacy or subgroup analyses are planned.

\section{Discussion}

\section{Strengths and limitations}

The proposed study is a rigorous single blind, two-arm RCT with a parallel group design to assess the efficacy of a knitting program, which is a low-cost, communitybased, innovative and accessible intervention at reducing hand impairment, improving occupational performance, as well as enhancing the self-efficacy, coping strategies and QoL of the older women with HOA $[5,6]$. The proposed knitting program is based on: 1) evidence stemming from physiological studies [34-36]; 2) recommendations for individuals over 50 years old with $\mathrm{OA}$ [37]) and specifically with HOA [12]; 3) evidence-based general exercise protocols for HOA $[5,38,39]$ and OA of the thumb [40-42]. The movements typically involved in hand therapy for HOA are also involved in knitting. The previous case study [43] and feasibility study conducted by co-investigators of this protocol suggest that knitting represents a promising, functional and meaningful activity for elderly women suffering from HOA to decrease morning stiffness and pain via its effect on biological, psychological and social factors [22]. The meaning attributed to this activity may contribute to increase adherence to the "exercise program". Optimal therapeutic and functional exercises dosage is difficult to select, especially considering that a study on knitting for
HOA has not been conducted as of yet [7]. A review of existing protocol $[5,38,39]$, and previous RCTs on therapeutic exercises $[9,14]$ were considered to inform the selection of the proposed knitting program intensity, frequency, duration. However, the existing protocols need to be adapted so they are tailored with to the therapeutic goals and knitting specificity. However, the knitting program was considered to be performed daily (not 2 to 3 days a week) since the immediate symptom effect lasts for four consecutive hours. Similarly to acetaminophen, knitting could be used as a temporarily pain or morning stiffness relief minus potential side effects of the drug often associated in adults [72]. However, it is hypothesized that regularity of any type of land-based exercise program seems more important than intensity to improve joint health [43]. A total study duration of 16 weeks (12-week knitting program plus a follow-up period of 4 weeks) is justified since it is not ethical to put a study participant on a long-term waiting list without an active intervention. The timeframe of the intervention and adherence in the long term beyond the 4-week follow-up won't be assessed. This compromise is mandatory in order to use a waitlist as control condition.

To minimize the potential misclassification bias, a rheumatologist involved as a co-investigator and specialized in HOA will make sure each participant enrolled in this RCT has a definite diagnostic of HOA. The ACR classification [50], the radiologic criteria according to [51], and the disease activity criteria according to the Doyle Articular Index [52] will be taken into consideration.

Assessments will include a range of clinical as well as implementation outcome measures. However, there is an increased risk of Type-1 error due to the presence of multiple outcomes. According to the adapted OMERACT group [73], all HOA studies should assess morning stiffness, pain, physical function, patient global assessment, joint activity and hand strength. In a recent systematic review [74], the AUSCAN, FIHOA, VAS pain, grip and pinch strength, and pain on palpation were the outcome measures most frequently used for HOA and provided supporting evidence for good metric properties [75-77].

Potential information bias includes the imprecision of self-reporting outcomes [78]. The inclusion of performancebased physical function measures (e.g. Functional Index for Hand OA (FIHOA) [69] rather than solely measuring self-reported measures (e.g. AUSCAN function subscale) [62, 63] would help address these limitations. The primary and secondary outcomes, respectively morning stiffness and pain, are self-reported, but represent important unwanted symptoms that have functional consequences that affect the QOL of individuals with HOA. Objective measures of these particular symptoms are difficult to obtain. However, pain and morning intensity using a visual analogue scale is 
recognized as a gold standard, since the nature of pain is a subjective perception/experience. Unfortunately, no technology can measure this personnel sensation outcome [79]. In addition to self-reported VAS measurements of pain and morning stiffness intensity before and after knitting, the number of squares knitted will be recorded in logs books to capture the knitting intensity/quantity. Daily self-reported VAS measurements of pain and morning stiffness intensity before and after knitting are important to be recorded in the participant's logbook, because these symptoms have a negative impact in their functional activities and QoL. Logbooks is a tool that can be useful to minimize potential recall biases. It might also be difficult to discriminate between pain from fingers versus from thumb for each hand assessment. This is why it important to take into consideration intensity, location, timing and duration of pain and joint stiffness [55] in the assessment in each finger affected by HOA.

This proposed RCT is necessary to address questions of clinical and scientific importance for rehabilitation specialists in improving the QoL of elderly women with HOA living in Canada. The results of this study will likely be generalizable to older women with mild to moderate HOA. If the results of this proposed RCT are positive, this accessible activity may be generalized to individual home-based knitting or group-based knitting in existing social clubs. The results of this RCT may give evidence to health professionals of an effective alternative physical activity to suggest for their patients with HOA.

\section{Conclusion}

This knitting RCT has substantial potential to enhance our understanding of this functional activity on the management of HOA, refine dosage and adherence recommendations in older women for this prevalent disease and to ultimately reduce the burden of disability in this aging population. This proposed RCT will contribute to the knowledge of the effect of knitting for individuals with HOA on self-reported and performance-based, HOA symptoms, level of QoL as well as fingers strength hand function and their self-efficacy and exercise adherence.

This proposed knitting RCT could also determine if this functional self-management activity has a daily and punctual effect on symptoms (i.e. HOA morning stiffness or pain) relief only (similarly to acetaminophen a pain reliever) or has also an effect on clinical and implementation outcomes,

This study could also make knitting the standard-ofcommunity-based-care for older women with HOA. Moreover, other rehabilitation or functional interventions could be examined for older people with other chronic diseases.

\section{Abbreviations}

ACR: American College of Rheumatology; ASES: Arthritis Self-Efficacy Scale; CHEO: Children Hospital of Eastern Ontario; CONSORT: Consolidated
Standards of Reporting Trials; DIP: distal interphalageal; FIHOA: Functional Index for Hand Osteoarthritis; HOA: Hand Osteoarthritis; HR: health-related; HSD: honest significance difference; ID: identification number; MCP: metacarpophalageal; OA: Ostoarthritis; OT: occupational therapist; PA: physical activity; PAR: Physical Activity Readiness; QoL: quality of life; RCT: randomized controlled trial; SPIRIT: Standard Protocol Items for Randomized Trials; VAS: visual analogue scale

\section{Acknowledgements}

Authors are indebted to members, knitting instructors and study subjects from the Pace Setters Seniors Club in Ottawa (Canada) who participated -in the feasibility study and to the Physiotherapy Foundation of Canada (not funded, but peer-reviewed) who provided very relevant feedback. The authors wish to offer a special thanks to C. Potvin for the bibliographical list and citations and to J. Taki, B. Desjardins and O. Thevenot who were involved as summer research assistants in the feasibility study data collection as well as E. Simoneau and A. Ferreira who helped with data entry of the participants' logbook information.

\section{Funding}

Support for the feasibility study was provided by grants University of Ottawa Research Chair Award for graduate students salary support (LB).

Availability of data and materials

Not applicable, this manuscript does not contain any data, since it is a protocol.

\section{Authors' contributions}

$P G, L B, N P, K T A, S C, G P, G L$ conceptualized the knitting intervention and conceptualized; PG, LB, GL conducted the feasibility study in Canada. PG, LB, KTA, GP, SZA established the selection criteria; GAW was in charge of the sample size calculation and data analyses conceptualization. GDA was instrumental in doing the legwork and initial draft of the grant that was accepted. All authors read and made comments on previous drafts of the manuscript, and approved the final manuscript.

\section{Ethics approval and consent to participate}

The study protocol is approved by the University of Ottawa Research Ethics Board (\#H02-16-12). All participants will sign an informed consent form before entering the study.

Consent for publication

Not applicable.

\section{Competing interests}

The authors declare that they have no competing interests.

\section{Publisher's Note}

Springer Nature remains neutral with regard to jurisdictional claims in published maps and institutional affiliations.

\section{Author details}

${ }^{1}$ School of Rehabilitation Sciences, Faculty of Health Sciences, University of Ottawa, 451 Smyth Road, Ottawa, ON K1H 8M5, Canada. ${ }^{2}$ School of Epidemiology, Public Health and Preventive Medicine, University of Ottawa, Ottawa, ON, Canada. ${ }^{3}$ The Arthritis Society, Ottawa Office, Ontario Division, Ottawa, ON, Canada. ${ }^{4}$ Children's Hospital of Eastern Ontario Research Institute, Department of Pediatrics, Faculty of Medicine and School of Rehabilitation Sciences, Faculty of Health Sciences, University of Ottawa, Ottawa, ON, Canada. ${ }^{5}$ School of Rehabilitation Sciences, University of Ottawa, Ottawa, ON, Canada. ${ }^{6}$ Ottawa Hospital, Ottawa, ON, Canada. ${ }^{7}$ Val-des-Monts, University of Sherbrooke; and researcher, Research Center on Aging,

Sherbrooke, QC, Canada.

Received: 20 June 2017 Accepted: 6 February 2018

Published online: 14 February 2018

\section{References}

1. Pereira D, Peleteiro B, Araújo J, Branco J, Santos RA, Ramos E. The effect of osteoarthritis definition on prevalence and incidence estimates: a systematic review. Osteoarthr Cartil. 2011;19:1270-85. 
2. Cho HJ, Morey V, Kang JY. Prevalence and risk factors of spine, shoulder, hand, hip, and knee osteoarthritis in community-dwelling Koreans older than age 65 years. Clin Orthop Relat Res. 2015;473:3307-14.

3. Haugen IK, Englund M, Aliabadi P, Niu J, Clancy M, Kvien TK, Felson DT. Prevalence, incidence and progression of hand osteoarthritis in the general population: the Framingham osteoarthritis study. Ann Rheum Dis. 2011;70(9):1-15.

4. Liu R, Damman W, Kaptein AA, Rosendaal FR, Kloppenburg M. Coping styles and disability in patients with hand osteoarthritis. Rheumatology. 2016;55:4118

5. Kjeken I, Grotle M, Hagen KB, Østerås N. Development of an evidence-based exercise programme for people with hand osteoarthritis. Scand J of Occup Ther. 2015;22(2):103-16.

6. Hill S, Dziedzic KS, Ong BN. The functional and psychological impact of hand osteoarthritis. Chronic Illness. 2010;6:101-10.

7. Østerås N, Hagen KB, Grotle M, Sand-Svartrud AL, Mowinckel P, Kjeken I. Limited effects of exercises in people with hand osteoarthritis: results from a randomized controlled trial. Osteoarthr Cartil. 2014a;22(9):1224-33.

8. Østerås N, Kjeken I, Smedslund G,Moe RH, Slatkowsky-Christensen B, Uhlig T, Hagen KB. Exercise for hand osteoarthritis. Cochrane database of systematic reviews 2017, issue 1. Art. No.: CD010388. DOI: 10.1002/ 14651858.CD010388.pub2

9. Stamm TA, Machold KP, Smolen JS, Fischer S, Redlich K, Graninger W, Ebner W, Erlacher L. Joint protection and home hand exercises improve hand function in patients with hand osteoarthritis: a randomized controlled trial. Arthritis \& Rheumatism. Arthritis Care \& Research. 2002;47(1):44-9.

10. Brosseau L, Wells GA, Tugwell P, Egan M, Dubouloz CJ, Casimiro L, Robinson V, Pelland L, McGowan J, Lamb M. Ottawa panel evidence-based clinical practice guidelines for therapeutic exercises and manual therapy in the treatment of osteoarthritis. Phys Ther. 2005a;85:907-71.

11. Rogers MW, Wilder FV. The effects of strength training among persons with hand osteoarthritis: a two-year follow-up study. J Hand Ther. 2007;20:244-9.

12. Zhang W, Doherty M, Leeb BF, Alekseeva L, Arden NK, Bijlsma JW, et al. EULAR evidence based recommendations for the management of hand osteoarthritis: report of a task force of the EULAR standing Committee for International Clinical Studies Including Therapeutics (ESCISIT). Ann Rheum Dis. 2007:66:377-38.

13. Lefler C, Armstrong J. Exercise in the treatment of osteoarthritis in the hands of the elderly. Clin Kinesiol. 2004;58:13-7.

14. Ye L, Kalichman L, Spittle A, Dobson F, Bennell K. Effects of rehabilitative interventions on pain, function and physical impairments in people with hand osteoarthritis: a systematic review. Arthritis Res Ther. 2011;13:R28.

15. Rogers MW, Wilder FV. Exercise and hand osteoarthritis symptomatology: a controlled crossover trial. J Hand Ther. 2009;22:10-7. https://doi.org/10.1016/ j.jht.2008.09.002

16. Hochberg MC, Altman RD, Toupin-April K, Benkhalti M, Guyatt G, Mcgowan J, Towheed T, Welch V, Wells GA, Tugwell P. American College of Rheumatology 2012 recommendations for the use of nonpharmacologic and pharmacologic therapies in osteoarthritis of the hand, hip, and knee. Arthritis Care \& Research Vol 64, No 4. 2012:46574. https://doi.org/10.1002/acr.21596.

17. Moe RH, Kjeken I, Uhlig T, Hagen KB. There is inadequate evidence to determine the effectiveness of nonpharmacological and nonsurgical interventions for hand osteoarthritis: an overview of high-quality systematic reviews. Phys Ther. 2009;89:1363-70. https://doi.org/10.2522/ptj.20080398.

18. Kjeken I, Smedslund G, Moe RH, Slatkowsky-Christensen B, Uhlig T, Hagen KB. Systematic review of design and effects of splints and exercise programs in hand osteoarthritis. Arthritis Care Res. 2011;63(6):834-48. https://doi.org/ 10.1002/acr.20427.

19. Valdes K, Marik T. A systematic review of conservative interventions for osteoarthritis of the hand. J Hand Ther. 2010;23:334-51.

20. Mahendira D, Towheed TE. Systematic review of non-surgical therapies for osteoarthritis of the hand: an update. Osteoarthr Cartil. 2009:17:1263-8.

21. Bement M, Weyer A, Hartley S, Yoon S, Hunter S. Fatiguing exercise attenuates pain-induced corticomotor excitability. Neurosci Lett. 2009:452:209-13.

22. Bement MH. Exercise-induced hypoalgesia: an evidence-based review. In: Sluka KA, editor. Mechanisms and Management of Pain for the Physical Therapist. Seattle: IASP Press; 2009. p. 143-66.

23. Koltyn KF. Exercise-induced Hypoalgesia and intensity of exercise. Sports Med. 2002;32(8):477-87.

24. Koltyn KF. Using physical activity to manage pain in older adults. J Aging Phys Act. 2002;10:226-39.
25. Regnaux JP, Lefevre-Colau MM, Trinquart L, Nguyen C, Boutron I, Brosseau L, Ravaud P. High-intensity versus low-intensity physical activity or exercise in patients with hip or knee osteoarthritis (Cochrane review). Cochrane database of systematic reviews (Online). 2015;10(2):CD010203.

26. Damush TM, Perkins SM, Mikesky AE, Roberts M, Motivational O'DJ. Factors influencing older adults diagnosed with knee osteoarthritis to join and maintain an exercise program. J Aging Phys Act. 2005;13:5-60.

27. Hoffman MD, Hoffman DR. Exercisers achieve greater acute exerciseinduced mood enhancement than nonexercisers. Arch Phys Med Rehabil. 2008:29:358-63.

28. Townsend EA, Polatajko HJ. Enabling occupation II: advancing an occupational therapy vision for health, well-being and justice through occupation. 2nd ed. Ottawa: CAOT; 2013.

29. Schutzer KA, Graves BS. Barriers and motivations to exercise in older adults. Prev Med. 2004;39:1056-61.

30. Krauss I, Katzmarek U, Rieger MA, Sudeck G. Motives for physical exercise participation as a basis for the development of patient-oriented exercise interventions in osteoarthritis: a cross-sectional study. European Journal of Physical and Rehabilitation Medicine. 2017; https://doi.org/10.23736/S19739087.17.04482-3.

31. Van Tuyl LHD, Boers M. Patient's global assessment of disease activity: what are we measuring? Arthritis Rheum. 2012;64(9):2811-3.

32. Stolee $P$, Rockwood K, Fox RA, Streiner DL. The use of goal attainment scaling in a geriatric care setting. J Am Geriatr Soc. 1992;40(6):574-8.

33. Lorig K, Brown BW Jr, Ung E, Chastain R, Shoor S, Holman HR. Development and evaluation of a scale to measure the perceived self-efficacy in people with arthritis. Arthritis Rheum. 1989;32(1):37-44.

34. Hoeger Bement MK, Dicapo J, Rasiarmos R, Hunter SK. Dose response of isometric contractions on pain perception in healthy adults. American College of Sports Medicine. 2008:40(11):1880-9.

35. Koltyn KF, Umeda M. Contralateral attenuation of pain after short-duration submaximal isometric exercise. J Pain. 2007:8(11):887-92.

36. Lemley KJ, Drewek B, Hunter SK, Hoeger Bement MK. Pain relief after isometric exercise is not task-dependent in older men and women. Journal of the American College of Sport. Medicine. 2014;46(1):185-91.

37. ACSM: American College of Sports Medicine, Pescatello LS. ACSM's guidelines for exercise testing and prescription. Philadelphia: Wolters Kluwer/Lippincott Williams \& Wilkins Health; 2014. p. 1456.

38. Østerås N, Risberg MA, Kvien TK, Engebretsen L, Nordsletten L, Schjervheim UB, Haugen I, Hammer HB, Provan S, Øiestad BE, Semb AG, Rollefstad S, Hagen KB, Uhlig T, Slatkowsky-Christensen B, Kjeken I, Flugsrud GB, Grotle M, Sesseng S, Edvardsen H, Natvig B. Hand, hip and knee osteoarthritis in a Norwegian population-based study - the MUST protocol. BMC Musculoskelet Disord. 2013;14:201-10. 1186/1471-2474-14-201

39. Østerås $N$, Hagen $K B$, Grotle $M$, Sand-Svartrud $A L$, Mowinckel $P$, Aas $\mathrm{E}$, Kjeken I. Exercise programme with telephone follow-up for people with hand osteoarthritis - protocol for a randomised controlled trial. BMC Musculoskelet Disord. 2014;15:82. https://doi.org/10.1186/14712474-15-82

40. Villafane $J H, C L E L A N D$ JA, Fernandez-De-Las-Penas C. The effectiveness of a manual therapy and exercise protocol in patients with thumb carpometacarpal osteoarthritis: a randomized controlled trial. J Orthop Sports Phys Ther. 2013;43(4):204-13.

41. Hamasaki T, Lalonde L, Harris P, Bureau NJ, Gaudreault N, Ziegler D, Choinière M. Efficacy of treatments and pain management for trapeziometacarpal (thumb base) osteoarthritis: protocol for a systematic review. BMJ Open. 2015;5:1-9.

42. Deveza LA, Hunter DJ, Wajon A, et al. Efficacy of combined conservative therapies on clinical outcomes in patients with thumb base osteoarthritis: protocol for a randomised, controlled trial (COMBO). BMJ Open. 2017;7: e014498. https://doi.org/10.1136/bmjopen-2016-014498.

43. Brosseau L, May LG. Knitting a promising pain self-management strategy for older woman with osteoarthritic hands? J Clin Rheumatol. 2017;23(3):179-80.

44. The Arthritis Society. 2009. Physical activity \& arthritis. http://www. webcitation.org/6PNKoYcT8.

45. Agha RZ, Douglas G, Altman DG, Rosin D. The SPIRIT 2013 statement: defining standard protocol items for trials. Int J Surg. 2015;13:288-91. https://doi.org/10.1016/j.ijsu.2014.12.007

46. Chan AW, Tetzlaff JM, Altman DG, et al. SPIRIT 2013 statement: defining standard protocol items for clinical trials. Ann Intern Med. 2013;158:200-7. 
47. Chan AW, Tetzlaff JM, Gøtzsche PC, et al. SPIRIT 2013 explanation and elaboration: guidance for protocols of clinical trials. BMJ. 2013;346:e7586.

48. Kloppenburg M, Maheu E, Kraus VB, Cicuttini F, Doherty M, Dreiser RL, Henrotin Y. OARSI clinical trials recommendations: design and conduct of clinical trials for hand osteoarthritis. Osteoarthr Cartil. 2015;23:772-86.

49. Schulz KF, Altman DG, Moher D. CONSORT 2010 statement: updated guidelines for reporting parallel group randomised trials. J Clin Epidemiol. 2010;63:834e840.

50. Altman R, Alarcon G, Appelrouth D, Bloch D, Borenstein D, Brandt K, Brown C, Cooke TD, Daniel W, Gray R, et al. The American College of Rheumatology criteria for the classification and reporting of osteoarthritis of the hand. Arthritis \& Rheumatism. 1990;33(11):1601-10.

51. Kallman DA, Wigley FM, JR SWW. New radiographic grading scales for osteoarthritis of the hand. Arthritis Rheum. 1989;32:1584-91.

52. Doyle DV, Dieppe PA, Scott J, Huskisson EC. An articular index for the assessment of osteoarthritis. Ann Rheumatic Dis. 1981;40:75-8.

53. Vliet Vlieland TP, Zwinderman AH, Breedveld FC, Hazes JM. Measurement of morning stiffness in rheumatoid arthritis clinical trials. J Clin Epidemiol. 1997; 50(7):757-63.

54. Brosseau L, Wells GA, Brooks S, De Angelis G, Bell M, Egan M, Poitras S, King J, Casimiro L, Loew L, Novikov M. People getting a grip on arthritis II: an innovative strategy to implement clinical practice guidelines for rheumatoid arthritis and osteoarthritis patients through Facebook. Health Educ J. 2014; 73(1):109-25.

55. Orbai AM, Halls S, Hewlett S, Bartlett SJ, Leong AL, Bingham CO 3rd, RA Flare Group Steering Committee. More than just minutes of stiffness in the morning: report from the OMERACT rheumatoid arthritis flare group Stiffneses breakout sessions. J Rheumatol. 2015;42(11):2182-4. https://doi. org/10.3899/jrheum.141172.

56. Scott J, Huskisson EC. Graphic representation of pain. Pain. 1976;2:175-84.

57. Oldfield RC. The assessment and analysis of handedness: the Edinburgh inventory. Neuropsychologia. 1971;9(1):97-113.

58. Veale JF. Edinburgh handedness inventory - short form: a revised version based on confirmatory factor analysis. Laterality. 2014;19:164-77.

59. Brosseau L, Wells G, Marchand S, Gaboury I, Stokes B, Morin M, Casimiro L, Yonge K, Tugwell P. Randomized controlled trial on low level laser therapy $(L L L T)$ in the treatment of osteoarthritis (OA) of the hand. Lasers Surg Med. 2005b;36:210-9.

60. Van Tulder MW, Assendett WJ, Koes BW, Bouter LM. Editorial Board of the Cochrane Collaboration Back Review Group. Method guidelines for systematic reviews in the cochrane collaboration back review group for spinal disorders: operationalization of van Tulder's quality assessment form. Spine. 1997;22:2323-30.

61. Garfinkel MS, Schumacher HR, Husain A, Levy M, Reshetar RA. Evaluation of a yoga based regimen for treatment of osteoarthritis of the hands. J Rheumatol. 1994;21:2341-3.

62. Bellamy N, Campbell J, Haraoui B, Buchbinder R, Hobby K, JC MD. Dimensionality and clinical importance of pain and disability in hand osteoarthritis: development of the Australian/Canadian (AUSCAN) osteoarthritis hand index. Osteoarthr Cartil. 2002;10:855-62.

63. Bellamy N, Campbell J, Haraoui B, Gerecz-Simon E, Buchbinder R, Hobby K, JC MD. Clinimetric properties of the auscan osteoarthritis hand index: an evaluation of reliability, validity and responsiveness. Osteoarthr Cartil. 2002b;10:863-9.

64. Bellamy N, Haraoui B, Buchbinder R, Hall S, Muirden K, Hobby K, Roth J, MacDermid JC, Soucy E, Gerecz-Simon E, Flynn J, Campbell J. Development of a disease-specific health status measure for hand osteoarthritis clinical trials: assessment of the symptom dimensionality. J Rheumatol. 1996;5:106S.

65. Sallis JF, Haskell WL, Wood PD, Fortmann SP, Rogers T, Blair SN, Paffenbarger RS. Physical activity assessment methodology in the Five-City project. Am J Epidemiol. 1985;121(1):91-106.

66. Sallis JF, Buono MJ, Roby JJ, Micale FG, Nelson JA. Seven-day physical activity recall. Med Sci Sports Exerc. 1997;29:89-103.

67. Hayden-Wade HA, Coleman KJ, Sallis JF, Armstrong C. Validation of the telephone and in-person interview versions of the 7-day PAR. Med Sci Sports Exerc. 2003;35(5):801-9.

68. Rabin R, de Charro F. EQ-5D: a measure of health status from the EuroQoL group. Ann Med. 2001;33(5):337-43.

69. Dreiser RL, Maheu E, Guillou GB, Caspard H, Grouin JM. Validation of an algofunctional index for osteoarthritis of the hand. Rev Rhum Engl Ed. 1995; 62(Suppl 1):43S-53S.
70. Murrock CJ, Abir Bekhet A, Zauszniewski JA. Psychometric evaluation of the physical activity enjoyment scale in adults with functional limitations. Issues in Mental Health Nursing. 2016;37(3):164-71. https://doi.org/10.3109/ 01612840.2015 .1088904

71. Tukey J. comparing individual means in the analysis of variance. Biometrics. 1949:5(2):99-114. JSTOR 3001913

72. Cipolat L, Loeb O, Latarche C, Pape E, Gillet P, Petitpain N. Acetaminophen: knowledge, use and overdose risk in urban patients consulting their general practitioner. A prospective, descriptive and transversal study Thérapie. 2017; 20(17):30036-7. https://doi.org/10.1016/j.therap.2016.12.012. [Epub ahead of print] pii: S0040-5957

73. Kloppenburg M, Bøyesen P, Visser AW, Haugen IK, Boers M, Boonen A, Conaghan PG, et al. Report from the OMERACT hand osteoarthritis working group: set of Core domains and preliminary set of instruments for use in clinical trials and observational studies. J Rheumatol. 2015;42(11):2190-7.

74. Willemien Visser A, Bøyesen P, Haugen IK, Schoones JW, van der Heijde DM, Rosendaal FR, Kloppenburg M. Instruments measuring pain, physical function, or Patient's global assessment in hand osteoarthritis: a systematic literature search. J Rheumatol. 2015;42(11):2118-34. https://doi.org/10.3899/ jrheum.141228.

75. Prodinger B, Stamm T, Peterson D, Stucki G, Tennant A. On behalf of the international classification of functioning, disability, and health info network. Toward a Standardized Reporting of Outcomes in Hand Osteoarthritis: Developing a Common Metric of Outcome Measures Commonly Used to Assess Functioning Arthritis Care \& Research. 2016;68(8):1115-27. https://doi. org/10.1002/acr.22816.

76. Taylor AM, Phillips K, Kushang K, Patel V. Assessment of physical function and participation in chronic pain clinical trials: IMMPACT/OMERACT recommendations. Pain. 2016;157:1836-50.

77. Dworkin RH, Turk DC, Farrar JT, Haythornthwaite JA, Jensen MP, Katz NP Kerns RD, Stucki G, Allen RR, Bellamy N, Carr DB, Chandler J, Cowan P, Dionne R, Galer BS, Hertz S, Jadad AR, Kramer LD, Manning DC, Martin S, McCormick CG, McDermott MP, McGrath P, Quessy S, Rappaport BA, Robbins W, Robinson JP, Rothman M, Royal MA, Simon L, Stauffer JW, Stein W, Tollett J, Wernicke J, Witter J. Core outcome measures for chronic pain clinical trials: IMMPACT recommendations. Pain. 2005;113:9-19.

78. Hartley S, Garland S, Young E, Bennell KL, Tay I, Gorelik A, Wark JD. A comparison of self-reported and objective physical activity measures in young Australian women. JMIR Public Health Surveill. 2015;1(2):e14

79. Marchand S. Le phénomène de la douleur. Les Éditions de la Chenelière inc: Montréal; 2009.

\section{Submit your next manuscript to BioMed Central and we will help you at every step:}

- We accept pre-submission inquiries

- Our selector tool helps you to find the most relevant journal

- We provide round the clock customer support

- Convenient online submission

- Thorough peer review

- Inclusion in PubMed and all major indexing services

- Maximum visibility for your research

Submit your manuscript at www.biomedcentral.com/submit 